Recepción: 17 / 04 / 2018

Aceptación: 03 / 05 / 2018

Publicación: 02 / 07 / 2018

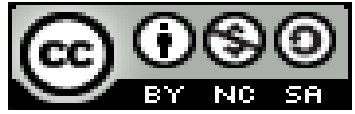

Ciencias técnicas y aplicadas

Artículo de Investigación

\title{
Diseño e implementación de un sistema electro-mecánico de selección de cambios de marcha en un vehículo de competencia para la Fórmula SAE
}

\section{Design and implementation of an electro-mechanical system for the selection of gear changes in a competitive vehicle for the SAE Formula}

\section{Projeto e implementação de um sistema eletromecânico para a seleção de mudanças de marchas em um veículo competitivo para a Fórmula SAE}

\author{
Luis F. Buenaño-Moyano ${ }^{\mathrm{I}}$ \\ lfbuenaño@espoch.edu.ec \\ Bolívar A. Cuaical-Angulo II \\ bcuaical@espoch.edu.ec Celin \\ A. Padilla-Padilla III \\ celin.padilla@espoch.edu.ec \\ Ligia E. Moreno-Pinduisaca IV \\ ligia.moreno@espoch.edu.ec \\ Ángel F. Pizarro-Torres v \\ angel.pizarro@espoch.edu.ec
}

Correspondencia: lfbuenaño@espoch.edu.ec

\footnotetext{
${ }^{I}$ Magister en Gestión del Mantenimiento Industrial, Ingeniero Mecánico, Facultad de Mecánica, Escuela Ingeniería Automotriz, Docente de la Escuela Superior Politécnica de Chimborazo ESPOCH, Riobamba, Chimborazo, Ecuador.

${ }^{\text {II }}$ Magister en Gestión de Energías, Ingeniero Automotriz, Docente de la Escuela Superior Politécnica de Chimborazo ESPOCH, Riobamba, Chimborazo, Ecuador.

III Magíster en Diseño Mecánico Mención en Fabricación de Autopartes de Vehículos, Master en Ingeniería de Vehículos Híbridos y Eléctricos, Diplomado Superior en Gestión del Aprendizaje Universitario, Ingeniero Automotriz, Facultad de Mecánica, Escuela Ingeniería Automotriz, Docente de la Escuela Politécnica de Chimborazo ESPOCH, Riobamba, Ecuador.

IV Magister en Gerencia y Liderazgo Educacional, Ingeniera Mecánica, Docente de la Escuela Politécnica de Chimborazo ESPOCH, Riobamba, Ecuador.

V Ingeniero Automotriz, Facultad de Mecánica, Docente de la Escuela Politécnica de Chimborazo ESPOCH, Riobamba, Ecuador.
} 


\section{Resumen}

El presente trabajo detalla el diseño y la implementación de un sistema electromecánico de selección de cambios de marcha en un vehículo de competencia para la Fórmula SAE. Para desarrollar el trabajo se aplicó una metodología de tipo explicativa, que permitió: establecer los parámetros de diseño del sistema electromecánico, calcular el momento de fuerza o torque necesario para mover la palanca de cambios aplicando el principio de funcionamiento de un dinamómetro, determinar los componentes del sistema electromecánico estructurado por los elementos de entrada/salida (sensores/servomotor) y la unidad de control que la constituye un Arduino Uno, construir el sistema electromecánico basado en los diagramas de conexión realizados en un software de simulación, verificar el óptimo funcionamiento del sistema en función del tiempo de accionamiento y el engrane correcto de cada una de sus marchas y analizar los resultados obtenidos por medio de simogramas y el método de Diseño Completamente al Azar. Una vez aplicada la metodología mencionada, los resultados obtenidos fueron: una reducción de 1.9 segundos del tiempo de accionamiento de la palanca de cambios y se mejoró el método de selección de cambios de marcha. Se concluyó que con la implementación del sistema electromecánico se redujo el tiempo de accionamiento de la palanca de cambios, se optimizó y se mejoró el método de conducción del vehículo. Finalmente se recomienda optimizar de la mejor manera la programación y los flujos de información, para evitar la saturación de los procesadores de la placa Arduino Uno y por ende lograr una mayor rapidez de funcionamiento.

Palabras clave: sistema electromecánico; cambio de marcha; accionamiento automático; palanca cambios; ingeniería del control automático.

\section{Abstract}

The present work details the design and implementation of an electromechanical system for selecting gear changes in a vehicle of competition for the SAE Formula. To develop the work, an explanatory methodology was applied, which allowed: to establish the design parameters of the electromechanical system, calculate the moment of force or torque needed to move the shift lever applying the operating principle of a dynamometer, determine the components of the electromechanical system structured by the input / output elements (sensors / servomotor) and the control unit that constitutes an Arduino Uno, build the electromechanical system based on the connection diagrams made in a simulation software, verify the optimal functioning of the system 
in function of the time of accionamiento and the correct gear of each one of its marches and to analyze the results obtained by means of simogramas and the method of Design Completely to the Chance. Once the aforementioned methodology was applied, the results obtained were: a reduction of 1.9 seconds of the time of activation of the shift lever and the method of selection of gear changes was improved. It was concluded that with the implementation of the electromechanical system the time of activation of the shift lever was reduced, the driving method of the vehicle was optimized and improved. Finally, it is recommended to optimize the programming and information flows in the best way, in order to avoid the saturation of the Arduino Uno processors and thus achieve a faster operation.

Keywords: Electromechanical system; gearshift; automatic drive; gear lever; automatic control engineering.

\section{Resumo}

O presente trabalho detalha o projeto e implementação de um sistema eletromecânico para a seleção de mudanças de marcha em um veículo de competição para a fórmula SAE. Para desenvolver o trabalho, aplicou-se uma metodologia explicativa, que permitiu: estabelecer os parâmetros de projeto do sistema eletromecânico, calcular o momento de força ou torque necessário para mover a alavanca de câmbio aplicando o princípio de funcionamento de um dinamômetro, determinar os componentes o sistema electromecânico estruturado elementos de entrada / saída (sensores / actuadores) e a unidade de controlo que é um Arduino uma construir o sistema electromecânico com base nos diagramas de ligação feitos em software de simulação, verificar a operação óptima sistema em função do tempo de accionamento e da marcha correta de cada uma de suas marchas e analisar os resultados obtidos por meio de simogramas e o método de Design Completely to the Chance. Uma vez aplicada a metodologia mencionada, os resultados obtidos foram: redução de 1,9 segundos do tempo de ativação da alavanca de câmbio e melhoria do método de seleção das mudanças de marcha. Concluiu-se que com a implementação do sistema eletromecânico o tempo de ativação da alavanca de troca foi reduzido, o método de condução do veículo foi otimizado e melhorado. Por fim, recomenda-se otimizar os fluxos de programação e informação da melhor maneira, a fim de evitar a saturação dos processadores Arduino Uno e assim alcançar uma operação mais rápida. 
Palavras chave: Sistema eletromecânico; mudança de marcha; acionamento automático; mudanças de alavanca; engenharia de controle automático.

\section{Introducción}

La Fórmula SAE conocida también como Fórmula Student es una competición organizada por la Society of Automotive Engineers, en la cual participan estudiantes de universidades de todo el mundo que diseñan, construyen y compiten con un vehículo monoplaza, en la que se ponen en práctica los conocimientos adquiridos por los estudiantes, al desafiarlos a diseñar, construir y probar el rendimiento y el desempeño de un vehículo real en un entorno competitivo a nivel mundial. Desde la carrera de Ingeniería Automotriz, de la Escuela Superior Politécnica de Chimborazo en aras de la competencia, los estudiantes se enfocaron en Para ello, se le instalo un sistema electro-mecánico de selección de cambios de marcha que le permita al vehículo reducir el tiempo de accionamiento de los cambios, lo cual, se detalla en el presente trabajo.

Para seleccionar las marchas del vehículo, el piloto acciona la palanca de cambios de forma tradicional, es decir, el piloto suelta una mano del volante y con esa mano acciona la palanca lo cual genera efectos negativos como: mayores tiempos de accionamiento (en relación al accionamiento automático), difícil acceso al mando de control de cambio de marcha, distracción del piloto al momento de conducir y disminuye la maniobrabilidad, seguridad y confortabilidad del piloto.

Para ello, hay que considerar que uno de los principales procesos que se realiza durante la conducción del vehículo es el de selección de marchas, por tal motivo, es importante estudiar a detalle dicho proceso con el fin de encontrar posibles mejoras y así obtener el máximo rendimiento del vehículo. En la que se considera que una alternativa, para mejorar el proceso de selección de marchas, es implementar un sistema electromecánico que optimice la selección de marchas y mitigue los efectos negativos (mencionados anteriormente) generados por el proceso tradicional, de selección de marchas, que realiza el piloto (Sanchez, 2004). Cabe destacar, que la aplicación de métodos y componentes que no se utilizan habitualmente pueden ser de mucha utilidad en el ámbito automotriz, tal es el caso del uso de un Arduino para el sistema electromecánico. 


\section{Consideraciones teóricas}

El Vehículo que entra en las competencias de Fórmula SAE debe ser concebido, diseñado, fabricado y mantenido por los miembros del equipo de estudiantes, sin la participación directa de los ingenieros profesionales, los ingenieros de automoción, pilotos, mecánicos o profesionales relacionados. (SAE, 2016)

En cuanto al chasis existen unas restricciones en relación al diseño y materiales empleados, sobre todo de cara a la seguridad del piloto. La mayoría de los equipos emplean chasis multitubulares de acero bajo en carbono. Para el apartado de los frenos hay mucha libertad con respecto al material empleado, siempre que sea un sistema hidráulico con dos circuitos independientes. (Motorcycle Safety Foundation, 2015).

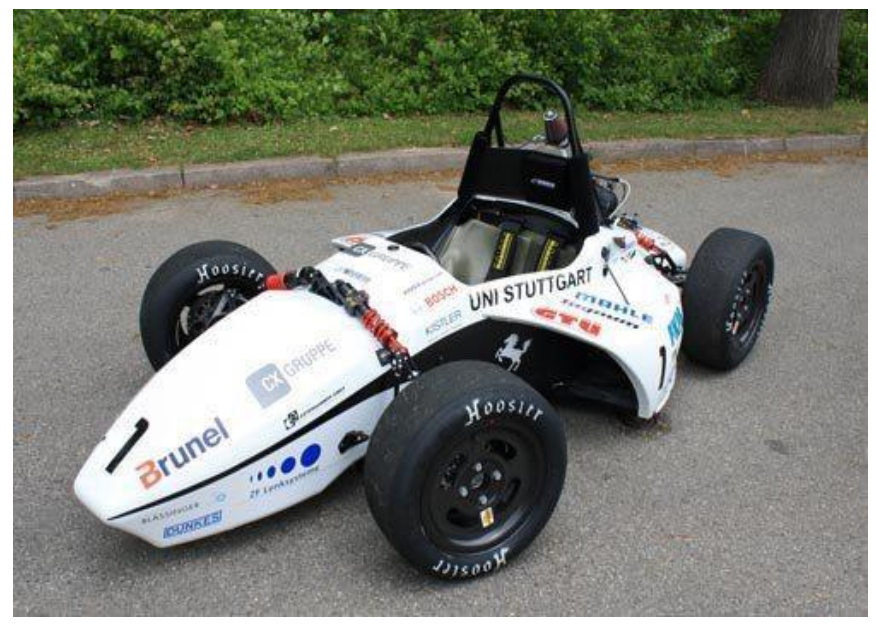

Figura 1. Vehículo monoplaza, fórmula SAE

\section{Sistema de transmisión}

El sistema de transmisión es el conjunto de elementos destinados a transmitir la potencia desde la salida del motor hasta las ruedas. Sus partes constitutivas cumplen tareas específicas y a su vez se interaccionan para trabajar en conjunto, contando con ubicaciones y cantidades variadas de acuerdo a la situación del motor y la configuración del eje motriz. (Taller Virtual, 2008)

En primer lugar, el embrague, situado entre el motor y la caja de cambios, acopla o desacopla el motor de la caja de cambios, al cambiar de marcha, o asegura una unión progresiva en el arranque. (Taller Virtual, 2008) 
La caja de cambios, situada entre el embrague y el eje motriz, modifica la fuerza o la velocidad aplicada a las ruedas, aprovechando la potencia del motor para vencer las resistencias del vehículo al desplazarse. El árbol de transmisión tiene la misión de transmitir el movimiento salido de la caja de cambios hacia el eje motriz, transmitiéndose así hasta las ruedas. (Taller Virtual, 2008). Finalmente, el eje motriz puede estar situado en la parte trasera, delantera o en ambas partes del vehículo, y tiene en su interior al par cónico piñón-corona que reduce la velocidad, y al diferencial, que permite que al tomar una curva la rueda exterior lleve más velocidad que la interior (relacionada con la diferencia de espacio recorrido). (Taller Virtual, 2008)

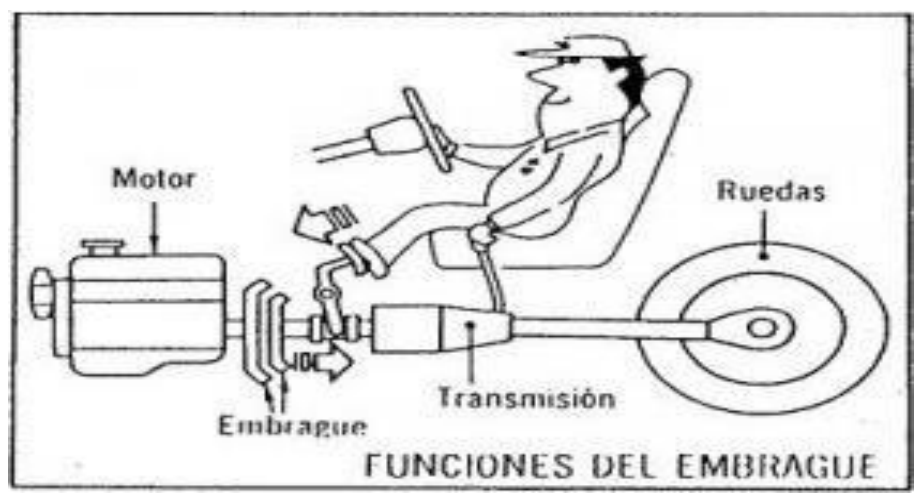

Figura 2. Sistema de transmisión. Recuperado en https://goo.gl/B45Qw8

\section{Caja de cambios de una motocicleta}

También conocida como caja de velocidades, es la encargada de transformar o mantener la relación de transmisión entre la salida del motor y el receptor de potencia hacia las ruedas. Está formada por varios grupos de engranes que al alternarse varían y transforman el par motor. (Gordillo Bravo, y otros, 2015)

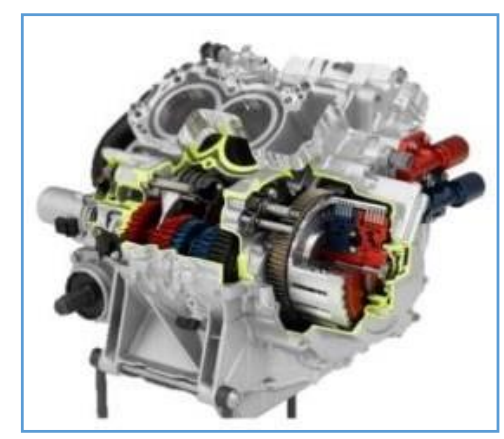

Figura 3. Compacto del motor y caja de cambios de una motocicleta. 


\section{Diseño completamente al azar}

Por medio de la implementación del sistema electromecánico se pretende optimizar el proceso de conducción del vehículo al reducir el tiempo de accionamiento de la palanca de cambios. Actualmente el accionamiento de la palanca de cambios se realiza de forma manual; con la adopción del sistema electromecánico, en la que el accionamiento de la palanca de cambios se realiza de forma automática. (Letran, 2013).

Con este antecedente; por medio de un diseño completamente al azar (DCA) se va a comprobar que el accionamiento automático es la mejor opción para optimizar el proceso de conducción del vehículo.

El diseño completamente al azar es una prueba estadística basada en el análisis de varianza, en donde la varianza total se descompone en la "varianza de los tratamientos" y la "varianza del error".

El objetivo del DCA es determinar si existe una diferencia significativa entre los tratamientos, para lo cual se compara si la "varianza del tratamiento" contra la "varianza del error" y se determina si la primera es lo suficientemente alta según la distribución F.

Las fórmulas que se aplican en el DCA se presentan en la siguiente tabla ANOVA (Análisis de varianza):

Tabla 1. ANOVA para el DCA

\begin{tabular}{|l|c|c|c|c|c|}
\multicolumn{1}{|c|}{$\boldsymbol{F V}$} & $\boldsymbol{S C}$ & $\boldsymbol{G L}$ & $\mathbf{C M}$ & $\boldsymbol{F}_{0}$ & Valor-p \\
\hline Tratamientos & $S C_{T R A T}=\sum_{i=1}^{k} \frac{Y_{i+1}^{2}}{n_{i}}-\frac{Y_{*}^{2}}{N}$ & $k-1$ & $C M_{T R A T}=\frac{S C_{T R A T}}{k-1}$ & $\frac{C M_{T R A T}}{C M_{E}}$ & $P\left(F>F_{0}\right)$ \\
Error & $S C_{E}=S C_{T}-S C_{T R A T}$ & $N-k$ & $C M_{E}=\frac{S C_{E}}{N-k}$ & & \\
Total & $S C_{T}=\sum_{i=1}^{k} \sum_{j=1}^{n_{i}} Y_{i j}^{2}-\frac{Y_{m}^{2}}{N}$ & $N-1$ & & & \\
\hline
\end{tabular}

Para facilitar el cálculo se utilizó el software "Minitab" en el cual solo se deben especificar las características del diseño.

Características del diseño. Se definen los tratamientos que se van a aplicar y la variable a medir. Para este caso, los tratamientos son el tipo de accionamiento: manual y automático y la variable a medir es el tiempo de accionamiento. Para concluir que existe diferencia entre el accionamiento 
manual y automático se debe comparar el coeficiente de Fisher "Fc" calculado de la tabla ANOVA con el coeficiente de Fisher que se presenta en tablas "Ft". Si Fc > Ft se concluirá que el accionamiento automático es la mejor opción para optimizar el proceso de conducción del vehículo. (Clemente, 2016).

\section{Materiales y métodos}

Para diseñar e implementar el sistema electromecánico de selección de marchas se siguió el siguiente procedimiento: a) Realizar un estudio de micromovimientos en el proceso de selección de marchas. b) Diseñar el sistema electromecánico, mediante la selección adecuada de elementos.

c) Construir el sistema electromecánico.

\section{Estudio de Micromovimientos}

El estudio de micromovimientos es una técnica que permite analizar, registrar y cronometrar el proceso de selección de cambios de marcha que realiza el piloto. El procedimiento consiste en:

- $\quad$ Realizar y analizar un video del proceso de selección de marcha.

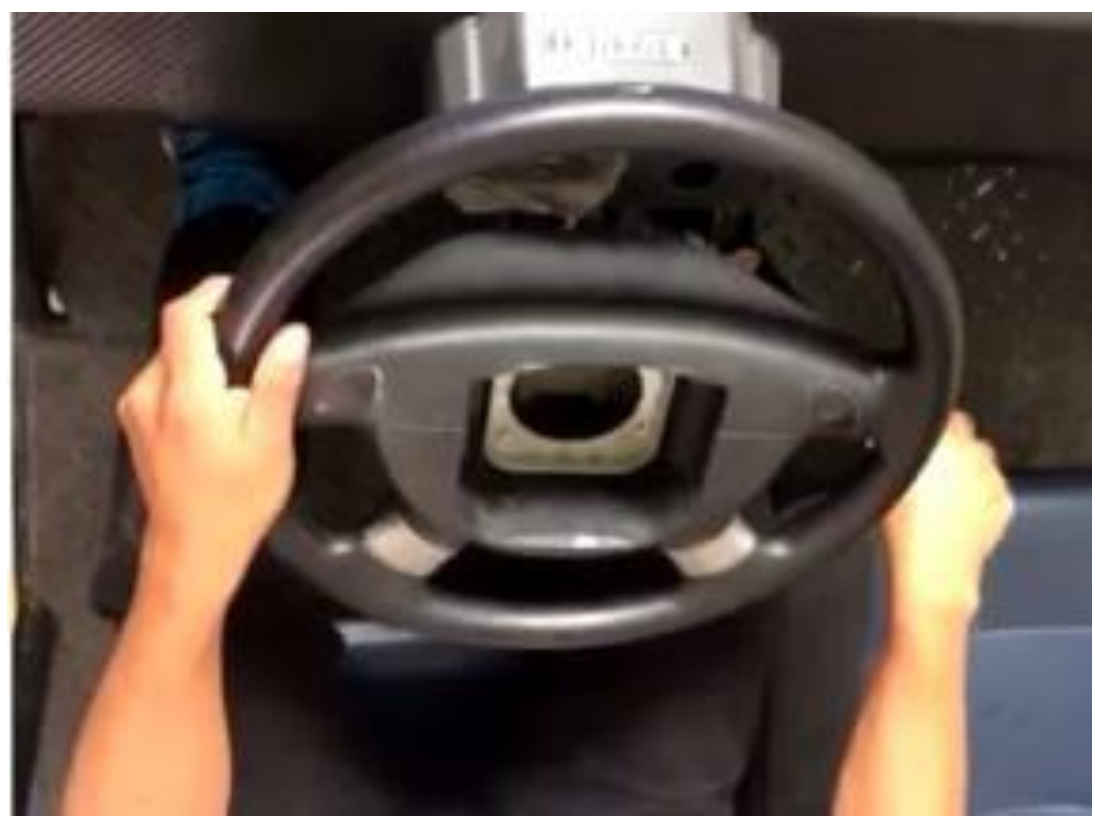

Figura 4. Proceso de selección de marcha

- $\quad$ Elaborar un simograma con los resultados del análisis. 
Tabla 2. Simograma, accionamiento manual

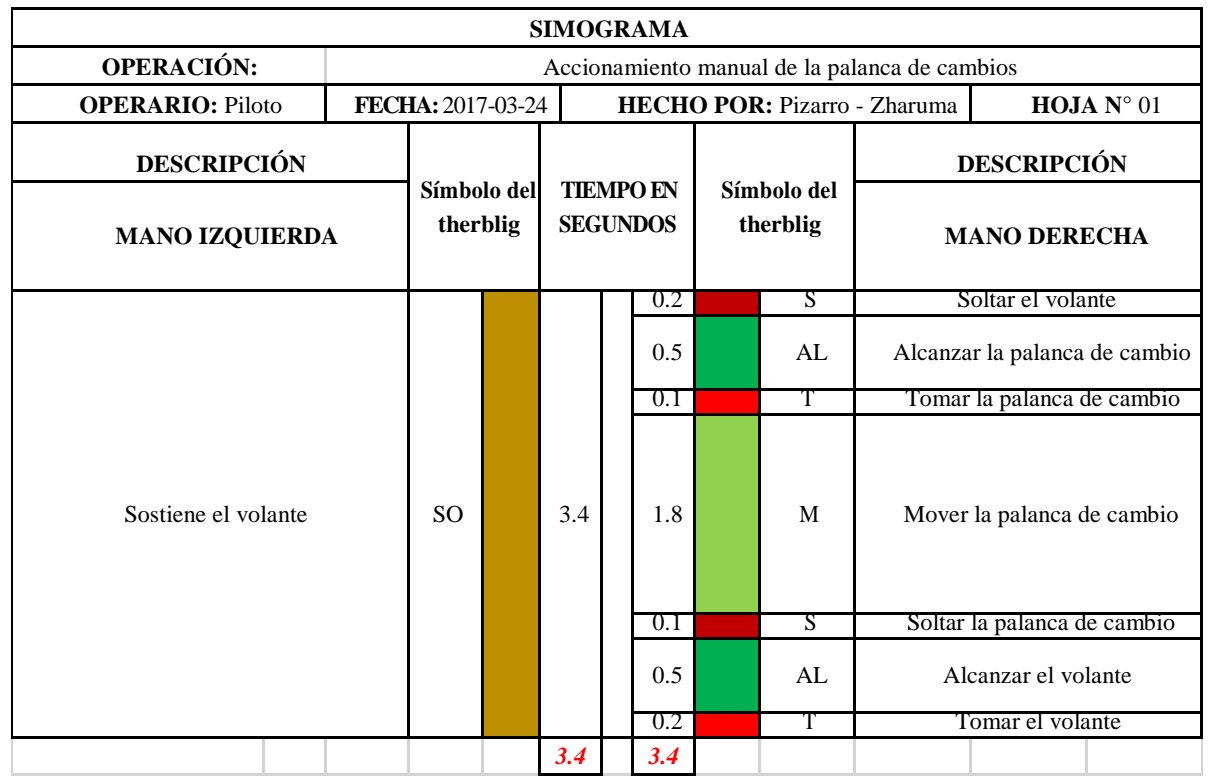

Según el simograma anterior, el tiempo que tarda un piloto para accionar una marcha manualmente es de 3.4 segundos aproximadamente.

- $\quad$ Desarrollar un mejor método para realizar el proceso de selección de marchas.

Como se puede observar en la figura 3, para seleccionar la marcha, el piloto realiza una serie de actividades que pueden sustituirse o incluso eliminarse por medio de la implementación de un sistema electromecánico.

Lo que se pretende lograr con el sistema electromecánico es que el piloto no suelte el volante para accionar la palanca de cambios, esto, con el fin de reducir el tiempo de accionamiento de la palanca de cambios, facilitar el acceso al mando de control de marcha, evitar la desconcentración del piloto al momento de la selección de marcha y aumentar la maniobrabilidad, seguridad y confortabilidad del piloto. Para hacer efectivo el objetivo planteado, se debe reemplazar el accionamiento manual de la palanca de cambios por un accionamiento automático. (Caiza y Arequipa, 2007).

\section{Diseño del sistema electromecánico}

Los sistemas electromecánicos surgen de la combinación de distintas áreas del conocimiento, como el electromagnetismo, la electrónica, la electricidad y la mecánica. Para el diseño del sistema electromecánico de selección de marchas se ha considerado la siguiente estructura: 
- Entradas: son sensores que introducen la información necesaria para el sistema y transforman una magnitud física en una señal eléctrica.

- Unidad de Control: procesa la información, enviada por los sensores de entrada, conforme a determinados procesos de cálculo matemáticos (algoritmos de control y regulación) y activan los elementos de salida o actuadores mediante señales eléctricas.

- Salidas: reciben las órdenes enviadas por la unidad de control y ejecutar tareas concretas. Transforman una corriente eléctrica de mando en movimiento, calor, luz, etc.

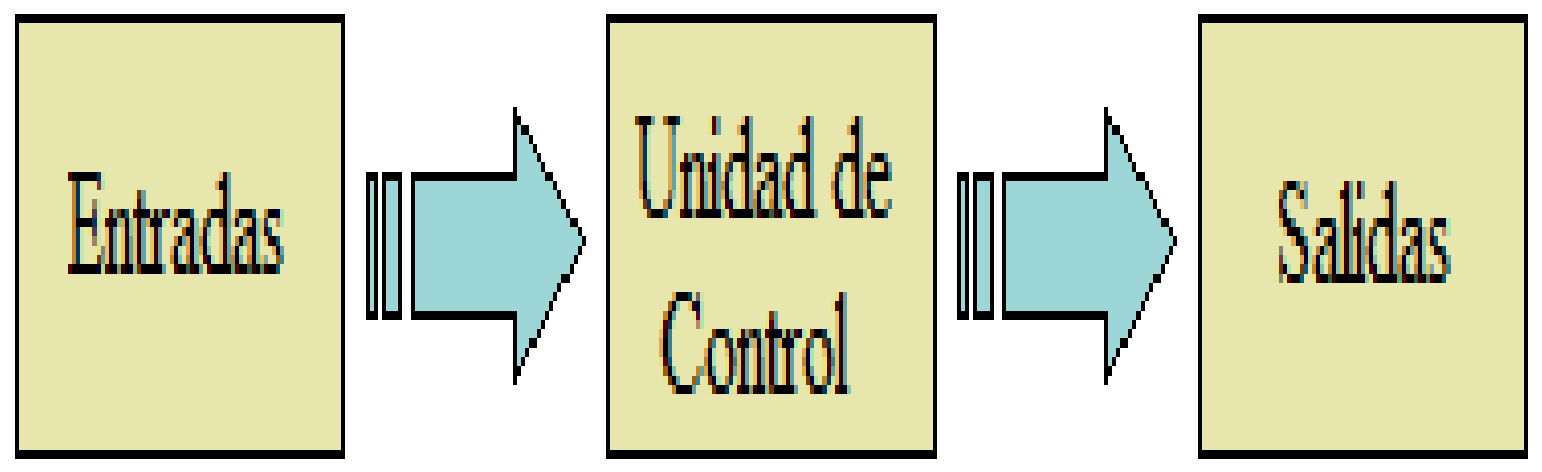

Figura 5. Estructura del sistema electromecánico

Los componentes que conforman cada bloque del sistema electromecánico son los siguientes:

Tabla 3. Componentes del sistema electromecánico

\begin{tabular}{|c|c|c|}
\hline Tipo de elemento & Elemento & Parámetro de selección \\
\hline Entradas & $\begin{array}{c}\text { - } 3 \text { Sensores de Contacto } \\
\text { (fines de carrera) KW11-3Z } \\
\text { - Switch carbono tipo avión } \\
\text { con led }\end{array}$ & $\begin{array}{c}\text { - Resistencia y facilidad de accionamiento } \\
\text { - Enclave de energización }\end{array}$ \\
\hline Unidad de Control & - Arduino & - Memoria y tamaño adecuado \\
\hline Salidas & $\begin{array}{l}\text { - Pantalla LCD } 1602 \\
\text { Controlador LCD I2C } \\
\text {-Servomotor } 50 \text { Kgf.cm }\end{array}$ & $\begin{array}{l}\text { - Fácil visualización que evite la distracción } \\
\text { - Fácil control de LCD } \\
\text { - Fuerza necesaria para el accionamiento }\end{array}$ \\
\hline $\begin{array}{l}\text { Elementos } \\
\text { Auxiliares }\end{array}$ & $\begin{array}{l}\text { Conductores Eléctricos } \\
\text { - Fuente regulada de voltaje } \\
\text { - Soporte para Arduino Uno } \\
\text { - Soporte para pantalla LCD }\end{array}$ & $\begin{array}{c}\text { - Resistencia ante esfuerzos } \\
\text { - Fuente con amperaje necesria para el correcto } \\
\text { funcionamiento } \\
\text { - Protección duradera y facilidad de montaje }\end{array}$ \\
\hline
\end{tabular}


Tabla 4. Descripción de los elementos de entrada

\begin{tabular}{|c|c|c|}
\hline \multicolumn{1}{|c|}{ Entrada } & Descripción \\
\hline $\begin{array}{l}\text { Sensores de Contacto } \\
\text { S1 reducir marcha } \\
\text { S2 incrementar marcha } \\
\text { S3 neutro }\end{array}$ & $\begin{array}{c}\text { Son dispositivos que al pulsarlos } \\
\text { envían señales a la unidad de } \\
\text { control para modificar la marcha } \\
\text { del vehículo. } \\
\text { Los sensores de contacto deben } \\
\text { acoplarse al volante. }\end{array}$ \\
\hline $\begin{array}{c}\text { Switch carbono tipo } \\
\text { avión con led }\end{array}$ & $\begin{array}{c}\text { Es un interruptor o dispositivo } \\
\text { que abre o cierra el paso de } \\
\text { corriente eléctrica al sistema } \\
\text { electromecánico. }\end{array}$ \\
\hline
\end{tabular}

Fuente: Autores

Tabla 5. Descripción de la unidad de control

\begin{tabular}{|c|c|c|}
\hline $\begin{array}{c}\text { Unidad de } \\
\text { Control }\end{array}$ & Descripción & Imagen \\
\hline Arduino Uno & $\begin{array}{l}\text { Es una placa electrónica basada en el } \\
\text { ATmega } 32 . \\
\text { Es el dispositivo que se encargará de } \\
\text { procesar las señales enviadas por el } \\
\text { sensor de contacto y actuar sobre las } \\
\text { salidas del sistema. }\end{array}$ & 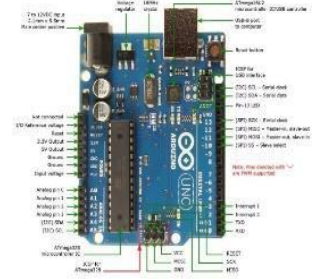 \\
\hline
\end{tabular}

Tabla 6. Descripción de los elementos de salida

\begin{tabular}{|c|c|c|}
\hline Salida & Descripción \\
\hline $\begin{array}{c}\text { Pantalla LCD } \\
1602\end{array}$ & $\begin{array}{c}\text { Es un dispositivo diseñado para } \\
\text { mostrar información en forma } \\
\text { gráfica. En este caso, mostrará la } \\
\text { marcha en el que se encuentra el } \\
\text { vehículo. }\end{array}$ \\
$\begin{array}{c}\text { Controlador LCD } \\
\text { I2C }\end{array}$ & $\begin{array}{c}\text { Es un dispositivo que nos permite } \\
\text { controlar una pantalla LCD a través } \\
\text { del bus I2C, usando únicamente dos } \\
\text { cables. }\end{array}$ \\
\hline
\end{tabular}




\begin{tabular}{|c|c|c|c|c|}
\hline Es un dispositivo que tiene la \\
capacidad de ubicarse en cualquier \\
posición dentro de su rango de \\
operación, y mantenerse estable en \\
dicha posición. \\
El servomotor se debe acoplar a la \\
palanca de cambios para que al \\
momento de girar accione la palanca.
\end{tabular} \mid

Tabla 7. Descripción de los elementos auxiliares

\begin{tabular}{|c|c|c|}
\hline Salida & Descripción & Imagen \\
\hline $\begin{array}{l}\text { Conductores } \\
\text { eléctricos }\end{array}$ & $\begin{array}{c}\text { Son hilos de metal (cobre o aluminio) } \\
\text { que se utilizan para conducir la corriente } \\
\text { eléctrica a través del sistema } \\
\text { electromecánico. }\end{array}$ & \\
\hline $\begin{array}{c}\text { Fuente regulada de } \\
\text { voltaje }\end{array}$ & $\begin{array}{c}\text { Permite ajustar voltajes en un } \\
\text { determinado rango según las } \\
\text { necesidades del sistema. } \\
\text { Se utilizará una fuente regulada de } \\
\text { voltaje 8A / 100W 12A Max DC 5-40V } \\
\text { a 1.2-36V. }\end{array}$ & \\
\hline
\end{tabular}

El funcionamiento del sistema electromecánico, se resume de la siguiente manera:

- Al presionar el sensor de contacto S1, el arduino ejecuta la orden de salida "reducir la marcha", el servo gira la palanca de cambios en sentido horario y la regresa a la posición inicial, el LCD muestra la marcha en la que se encuentra el vehículo.

- Al presionar el sensor de contacto S2, el arduino ejecuta la orden de salida "incrementar la marcha", el servo gira la palanca de cambios en sentido antihorario y la regresa a la posición inicial, el LCD muestra la marcha en la que se encuentra el vehículo.

- Al presionar el sensor de contacto S3, el arduino ejecuta la orden de salida "regresar a neutro", el servo gira la palanca de cambios en sentido horario y la regresa a la posición inicial (repite este proceso hasta alcanzar el neutro). 
La conexión de los elementos de entrada y salida a la unidad de control se detalla en la siguiente figura.

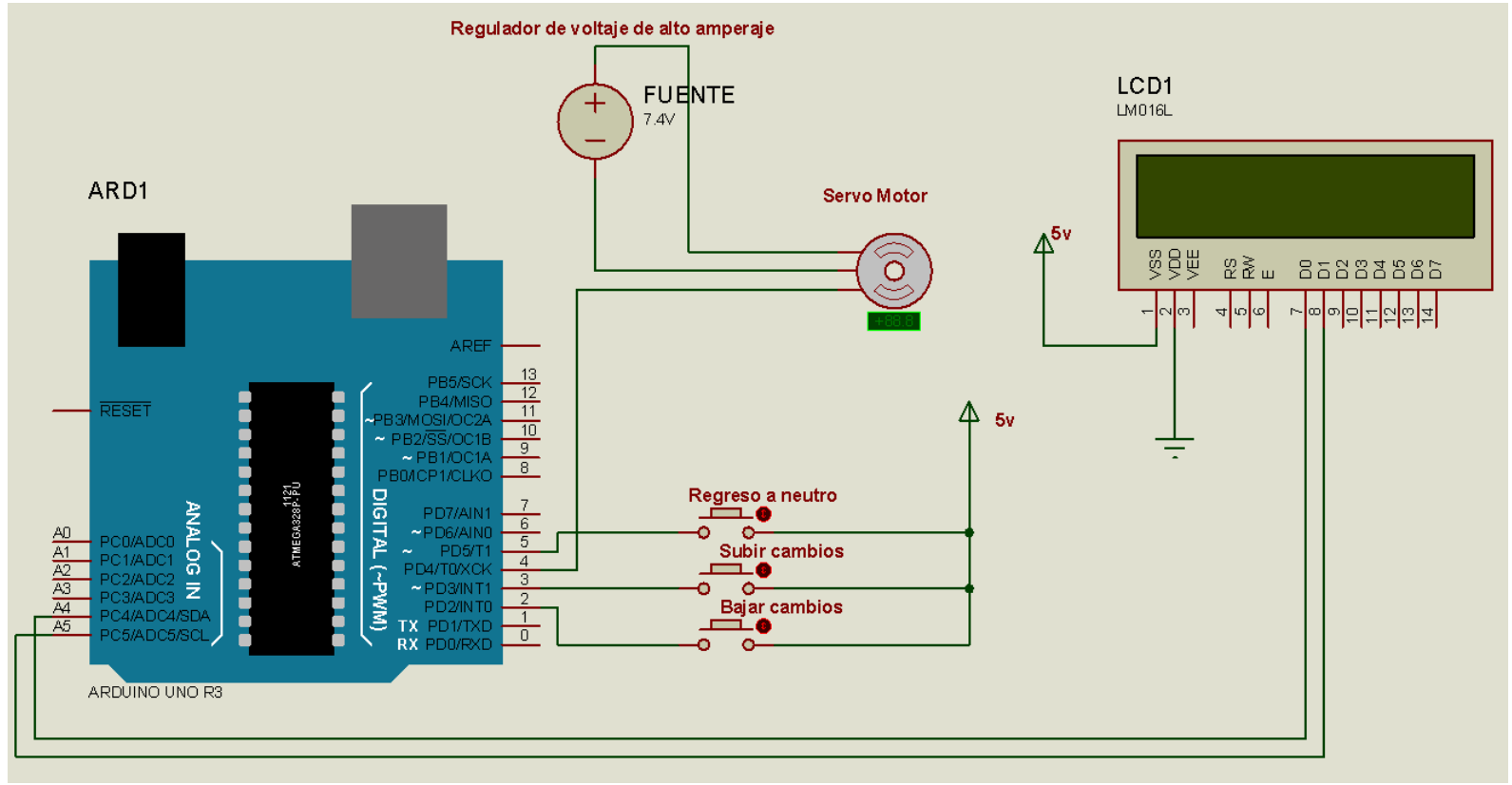

Figura 6. Diagrama de conexión del sistema electromecánico

\section{Resultados}

\section{Construcción del sistema electromecánico}

- Se realizó la conexión del sistema electromecánico tal como se detalla en la Figura 6, luego se procedió a ubicar en el lugar más adecuado cada elemento del sistema. Para facilitar la conexión de las entradas y salidas en la unidad de control, se utilizó borneras electrónicas. La unidad de control se ubicó debajo del panel de control del vehículo.

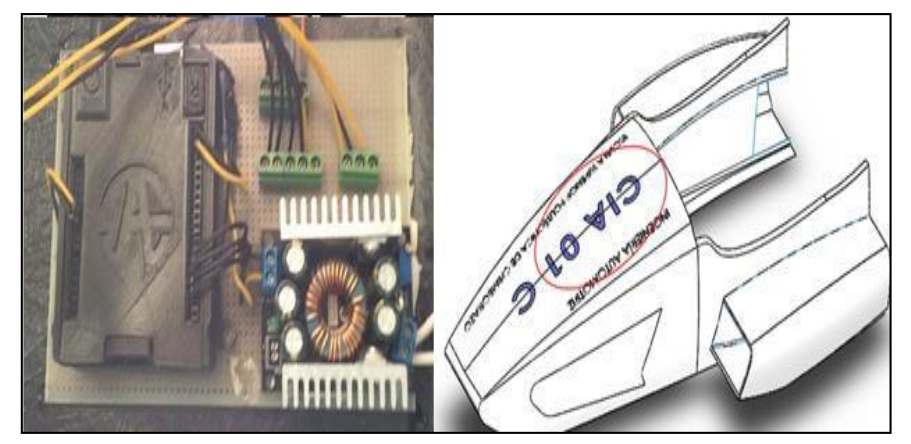

Figura 7. Conexión de las entradas y salidas a la unidad de control 
- El switch o interruptor se conectó, por medio de bornes, a la batería del vehículo; la cual proporciona la energía necesaria para el funcionamiento óptimo del sistema.

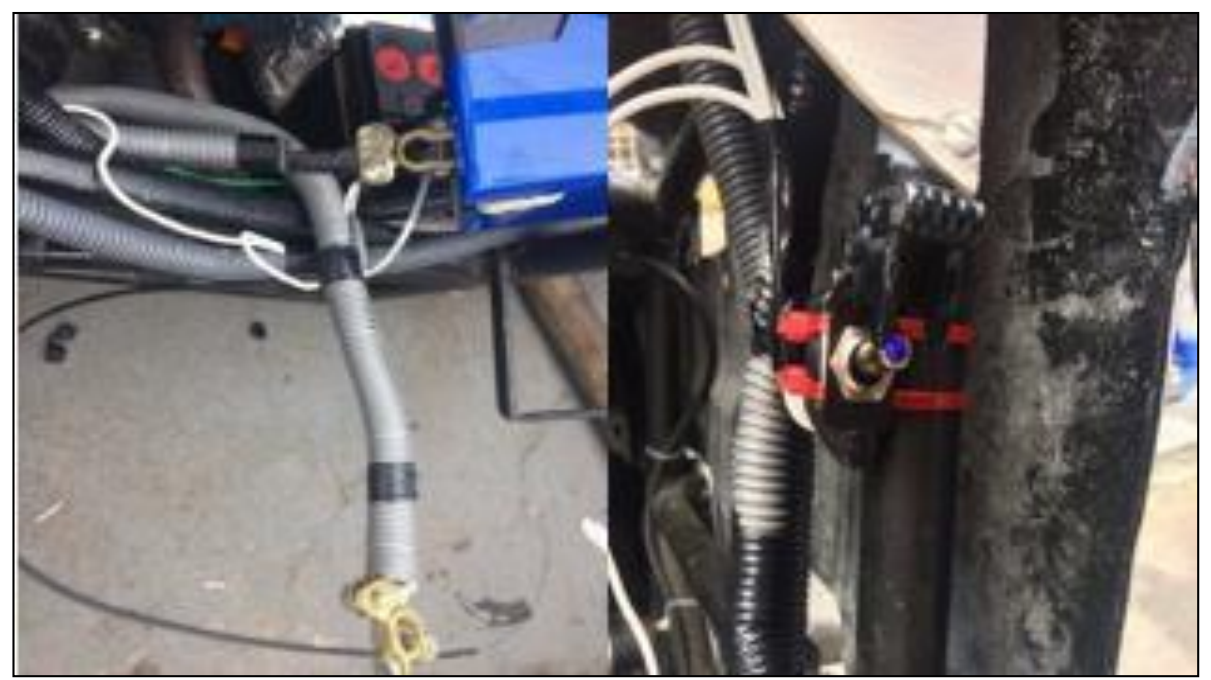

Figura 8. Instalación del interruptor

- La pantalla LCD se instaló junto a la pantalla principal del panel de control del vehículo; gracias a esta ubicación el conductor puede visualizar fácilmente la marcha en la que se encuentra el vehículo.

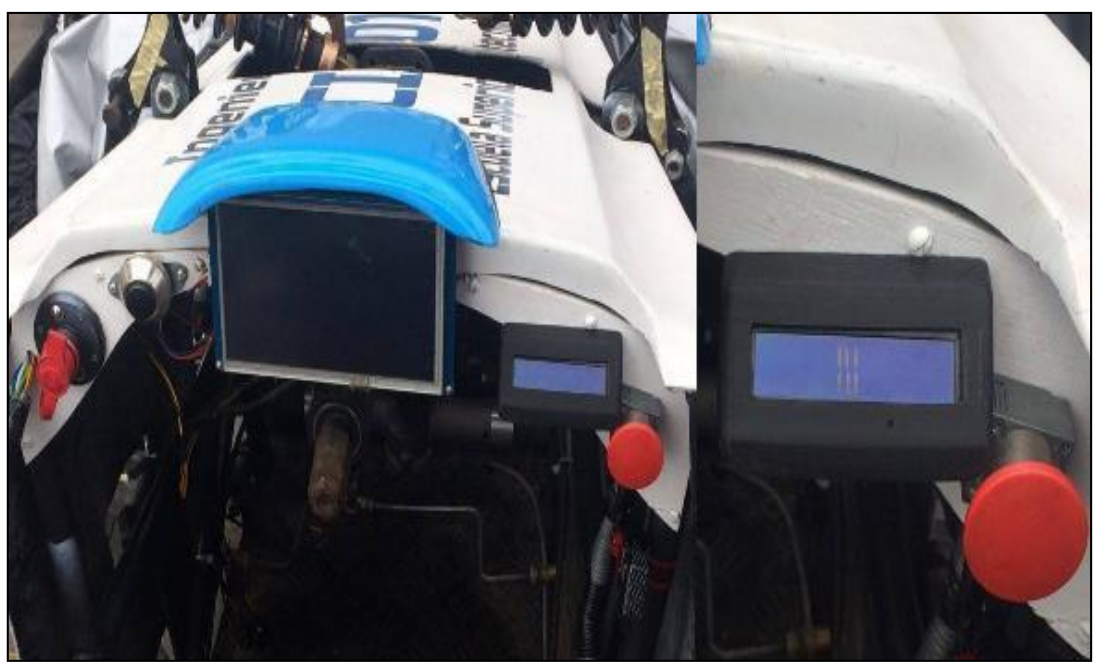

Figura 9. Instalación de la pantalla LCD

- El servomotor se instaló debajo de la palanca de cambios del vehículo; para la instalación del servomotor se diseñó una base que posicionara y sujetara al servo. 


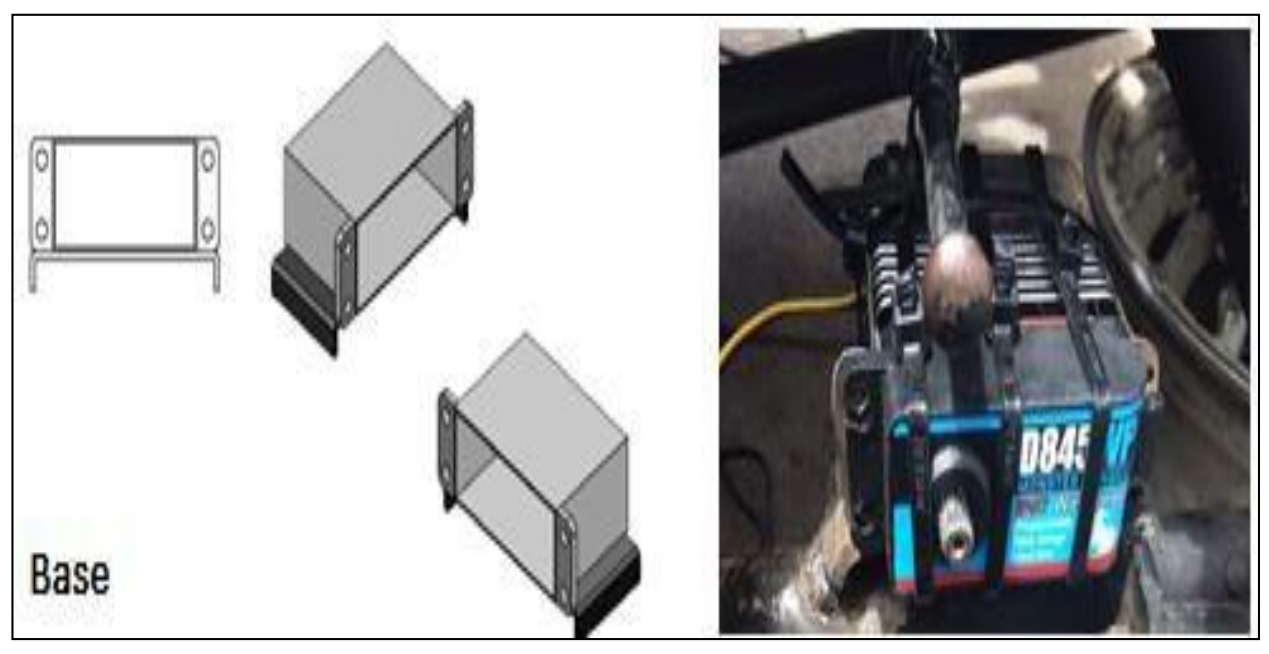

Figura 10. Instalación del servo

Para el acople del servomotor a la palanca de cambios se diseñó la siguiente pieza:

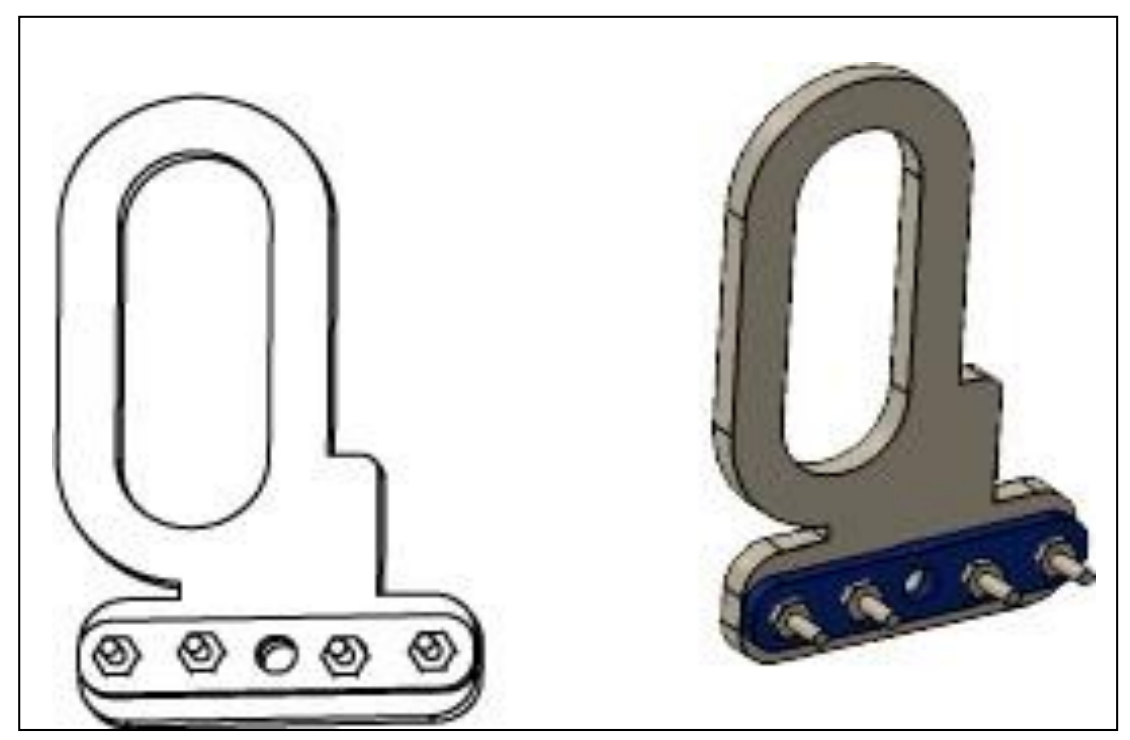

Figura 11. Acople del servo y palanca

Para el ensamble del servo y la palanca de cambios se utilizó rodamientos que facilitarán el giro del servo con la palanca de cambios. El ensamble se muestra en la figura 11. 


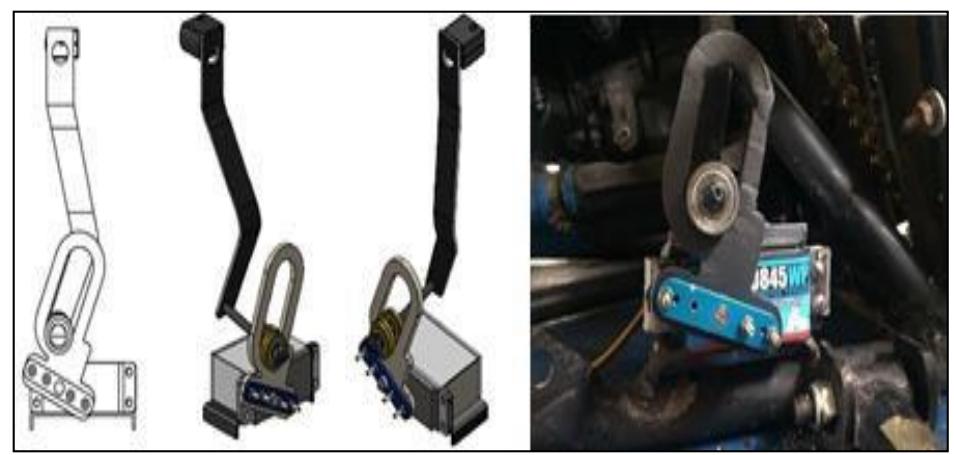

Figura 12. Ensamble servomotor y palanca de cambios

- Para instalar los sensores de contacto en el volante, se diseñó un acople desmontable. El diseño consta de 3 elementos en forma de paletas para que al momento de presionarlos se accione el sensor de contacto deseado, dicho acople se monta en la parte posterior del volante.

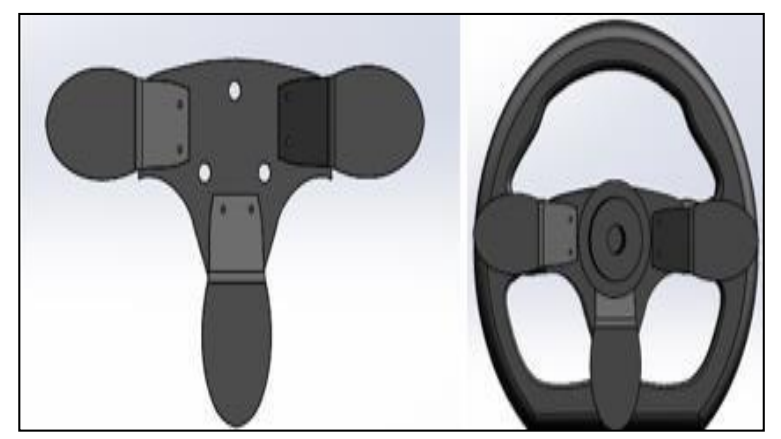

Figura 13. Acople para los sensores de contacto

Los sensores de contacto van ubicados debajo de las paletas como se muestra en la figura 14.
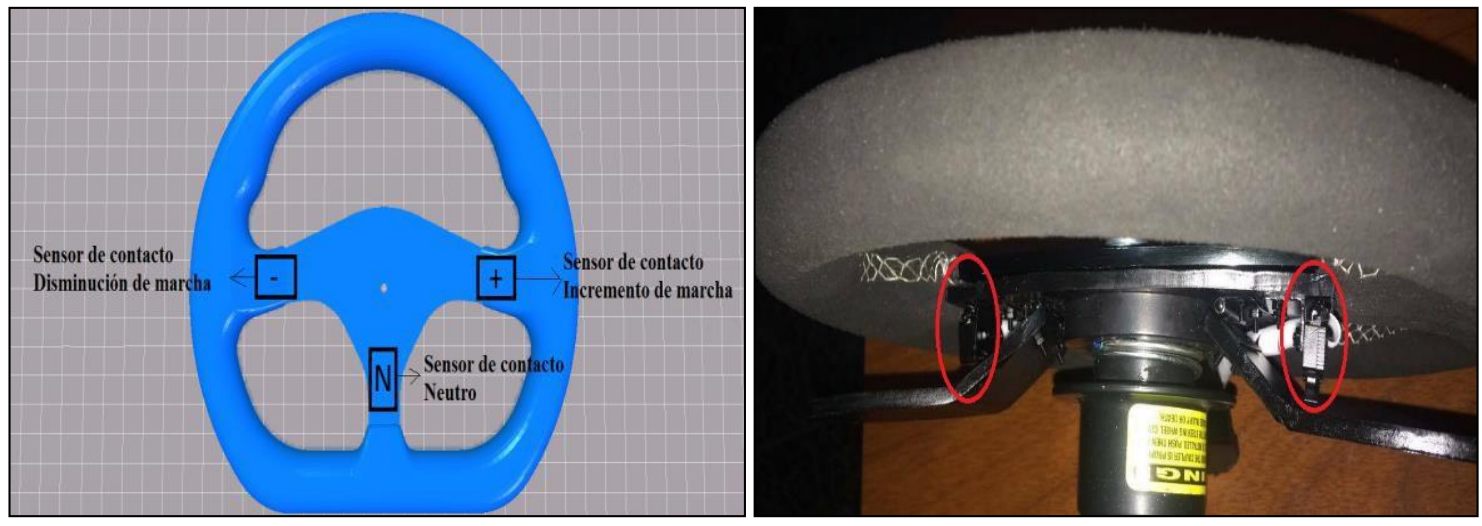

Figura 14. Instalación sensores de contacto 


\section{Resultados}

Se comprobó el funcionamiento del sistema electromecánico y los resultados obtenidos fueron satisfactorios. Los resultados se midieron en función del tiempo de accionamiento del sistema electromecánico y el engrane correcto de cada una de sus marchas.

Se realizaron 3 pruebas, la primera con la paleta del lado izquierda la cual acciona el aumento de marchas; la segunda prueba con la paleta derecha para la disminución de marcha y la tercera y última la paleta del medio la cual disminuye las marchas hasta el neutro identificando la marcha en la que se encuentre.

En la siguiente tabla se muestra los tiempos en segundos de accionamiento obtenidos:

Tabla 8. Tiempos de accionamiento en segundos

\begin{tabular}{|c|c|c|c|c|c|c|c|c|c|c|c|c|c|c|c|c|c|c|}
\hline \multirow{2}{*}{ 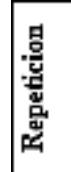 } & \multicolumn{6}{|c|}{$\begin{array}{l}\text { Prueba } 1 \\
\text { Marcha }\end{array}$} & \multicolumn{6}{|c|}{$\begin{array}{c}\text { Prueba } 2 \\
\text { Marcha }\end{array}$} & \multicolumn{6}{|c|}{$\begin{array}{l}\text { Prueba } 3 \\
\text { Marcha }\end{array}$} \\
\hline & $\vec{z}$ & I & m & $\underset{m}{ \pm}$ & $\hat{f}$ & 9 & $\hat{b}$ & 志 & m & ले & $\vec{d}$ & 罕 & 晕 & 鬲 & 䜆 & 同 & 乭 & 㫘 \\
\hline 1 & 1,20 & 1,00 & 1,03 & 1,05 & 1,04 & 1,06 & 1,05 & 1,01 & 0,98 & 1,03 & 1,01 & 1,10 & 1,22 & 1,65 & 2,05 & 2,36 & 2,80 & 3,20 \\
\hline 2 & 1,18 & 1,05 & 1,01 & 0,95 & 1,01 & 0,99 & 1,01 & 1,06 & 1,05 & 0,94 & 0,95 & 1,04 & 1,20 & 1,60 & 2,00 & 2,40 & 2,75 & 3,17 \\
\hline 3 & 1,23 & 0,96 & 0,97 & 1,02 & 0,98 & 1,00 & 0,94 & 1,03 & 1,02 & 1,00 & 1,02 & 1,22 & 1,18 & 1,55 & 1,97 & 2,44 & 2,84 & 3,23 \\
\hline 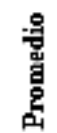 & 1,20 & 1,00 & 1,00 & 1,00 & 1,01 & 1,01 & 1,00 & 1,03 & 1,01 & 0,99 & 0,99 & 1,12 & 1,20 & 1,60 & 2,00 & 2,40 & 2,79 & 3,20 \\
\hline
\end{tabular}

Fuente: Elaboración propia

El mecanismo de accionamiento de las marchas, tanto en el incremento y disminución, son similares por lo tanto se calculó un promedio por repetición.

Promedio de repetición $1=1,4$ segundos

Promedio de repetición $2=1,2$ segundos

Promedio de repetición 3=1,3 segundos

En definitiva, el tiempo que tarda el piloto para accionar una marcha, por medio del sistema electromecánico, es de 1.2 a 1.4 segundos. 


\section{Discusión}

En la figura 3, se observa que para seleccionar la marcha, el piloto realiza una serie de actividades que pueden sustituirse o incluso eliminarse gracias a la implementación del sistema electromecánico de selección de marchas.

El sistema reemplaza el accionamiento manual de la palanca de cambios por un accionamiento automático. Gracias a esto el piloto no tiene la necesidad de soltar el volante a la hora de accionar la marcha, por lo cual, se reduce el tiempo de accionamiento de la palanca de cambios, se facilita el acceso al mando de control de marcha, se evita la desconcentración del piloto al momento de la selección de marcha y se aumenta la maniobrabilidad, seguridad y confortabilidad del piloto (Bestraten, 1994). Este hecho se puede evidenciar en el simograma de la figura 14.

Tabla 9. Simograma, Accionamiento automático

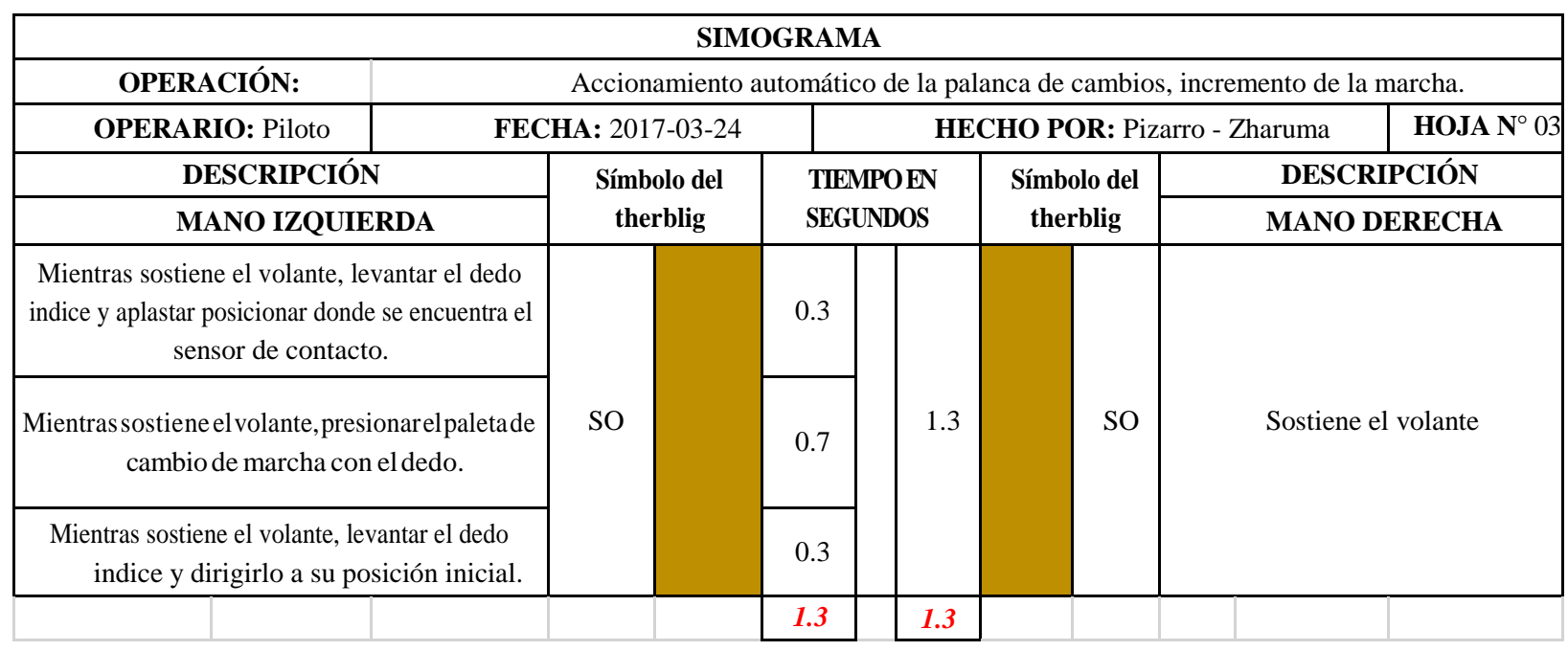

Para comprobar que el accionamiento automático es la mejor opción para optimizar el proceso de conducción del vehículo se compara los dos tipos de accionamiento, manual y automático. Para ello, se han registrado diferentes tiempos que tarda el conductor en accionar la palanca de cambios: manualmente y automáticamente. Los resultados obtenidos se muestran en la siguiente tabla: 
Tabla 10. Tiempos de accionamiento

\begin{tabular}{|c|c|c|}
\cline { 2 - 3 } \multicolumn{1}{c|}{} & \multicolumn{2}{c|}{ Tiempo (segundos) } \\
\hline Pruebas & Accionamiento Manual (M) & Accionamiento Automático (A) \\
\hline 1 & 3,2 & 1,4 \\
\hline 2 & 3,4 & 1,2 \\
\hline 3 & 3,0 & 1,3 \\
\hline
\end{tabular}

Fuente: Elaboración propia

El tiempo promedio del accionamiento manual y automático es:

$$
\begin{aligned}
& { }_{T_{\bar{W}}} \sum \frac{T_{M}}{3}=\frac{T_{1}+T_{2}+T_{3}}{3}={ }^{3.2+3.4+3}=3.2 \mathrm{~s} \\
& { }_{T_{\bar{A}}=} \sum \frac{T_{A}}{3}=\frac{T_{1}+T_{2}+T_{3}}{3}=\frac{1.4+1.2+1.3}{3}-1.3 \mathrm{~s}
\end{aligned}
$$

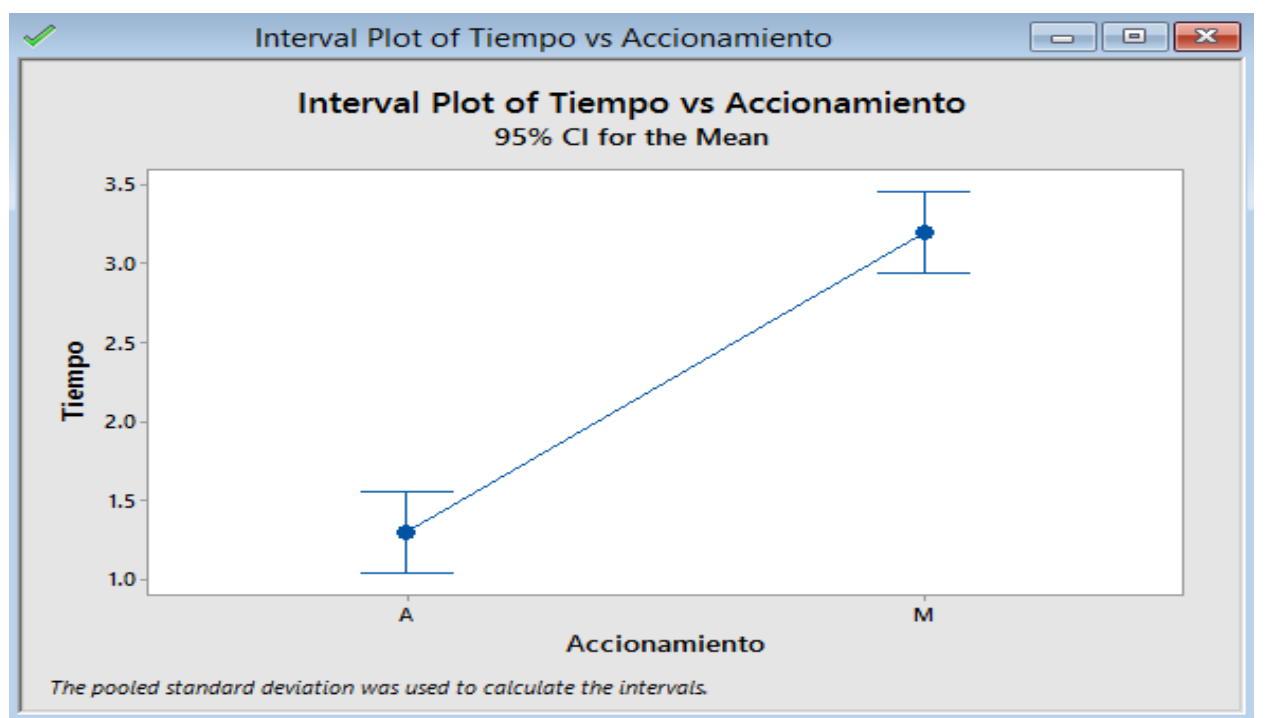

Figura 15. Comparación de los tiempos promedio

Como se puede observar en el gráfico, el tiempo promedio de accionamiento automático es menor que el tiempo promedio de accionamiento manual. Por lo tanto, se concluye que el accionamiento automático es la mejor opción para la optimización del proceso de conducción del vehículo con un tiempo promedio de accionamiento de $1.3 \mathrm{~s}$.

$$
{ }^{-} T_{M}^{-}{ }^{-} T_{A}^{-}=3.2-1.3=1.9 \mathrm{~s}
$$

El sistema electromecánico reduce el tiempo de accionamiento de la palanca de cambios un total de 1.9 segundos. 


\section{Conclusiones}

Se diseñó y construyó el sistema electromecánico de cambios de marchas mediante la selección adecuada de elementos, el sistema cumple con los parámetros de diseño y optimiza el proceso de conducción del vehículo.

El sistema electromecánico de cambios de marcha redujo el tiempo promedio de accionamiento de la palanca de cambios un total de 1,9 segundos.

Los elementos de control del sistema electromecánico están diseñados para ser ubicados en el volante del vehículo, esto facilita el acceso al mando de control de marcha.

Con la implementación del sistema electromecánico de cambios de marcha en el vehículo, el piloto evita desconcentraciones al momento de accionar la marcha; pues estas son accionadas sin que el piloto tenga que perder contacto con el volante.

Al ubicar los elementos de control del sistema electromecánico en el volante, el piloto no pierde contacto con el volante al momento de accionar la marcha por ende se aumenta la maniobrabilidad del vehículo y se mejora la confortabilidad (ergonomía) del piloto.

Se comprobó el funcionamiento de los elementos del sistema electromecánico; probando que los sensores de contacto envíen correctamente las señales de entrada, que el arduino procese debidamente la programación y que el servo y la pantalla LCD ejecuten correctamente las órdenes enviadas por la unidad de control; de esta forma el sistema fue probado y calibrado con satisfacción.

\section{Referencias Bibliográficas}

Arduino. (2017). Arduino uno open soft. [En línea]. [Consulta: 18 de enero de 2017.] Diponible en:http://www.arduino.org/products/boards/arduino-uno.

Bestraten, M. (1994). Ergonomía. Barcelona: INSHT. pp 13-18

Caiza, P y Arequipa, O (2007). Diseño y construcción de un mecanismo para automatizar el sistema de selección de velocidades para el vehículo corsa evolution 1400 cc (tesis). Escuela Politécnica Del Ejército. Latacunga-Ecuador 2007. pp-44-47. [Consulta: 28 de junio de 2017]. Disponible en: http://dspace.espoch.edu.ec/handle /123456789/655678 
Clemente, F (2016). Fase en español. [En línea] 2016. pp 46-48. [Consulta: 23 de febrero de 2017]. Disponible en: http://fsaenespanol.blogspot.com/2016/05/normas-formula-sae-2017-2018.html.

Bravo, G; Xavier E y Lara, Luis (2015). Diseño e implementación de un cambiador de marchas inteligente utilizando sistemas fpga para el prototipo FESPE 2013. Escuela Politécnica Del Ejército. Latacunga-Ecuador. 2015. pp-44-47. [Consulta: 28 enero de 2017]. Diponible en: http://dspace.espoch.edu.ec/handle/1234564589/6589

Letrán, J (2013). Corto Circuito. [En línea]. [Consulta: 18 de febrero de 2017]. Diponible en:http://www.cortoc.com/2011/12/introduccion-arduino.html.

MOTORCYCLE SAFETY FOUNDATION. (2015) Manual del operador de motocicleta. USA: Impresión y distribución cortesía de MSF, 2014. pp 45-55

Sanchez, V. Cajas Automáticas. En línea] 2004. [Consulta: 8 de febrero de 2017.] Disponible en:https://motorycompeticion.wordpress.com/descargas/.

TALLER VIRTUAL. (2008). Actualidad motor. [En línea]. [Consulta: 8 de marzo de 2017]. Disponible en: https://motorycompeticion.wordpress.com/descargas/. https://www.actualidadmotor.com/partes-constitutivas-de-los-sistemas-de-transmision/. 